\title{
Evaluation of cystamine-modified hyaluronic acid/chitosan polyplex as retinal gene vector
}

\author{
Ana V. Oliveira ${ }^{\mathrm{a}, \mathrm{b}}$, Adriana Marcelo ${ }^{\mathrm{c}}$, Ana M. Rosa da Costa ${ }^{\mathrm{d}, \mathrm{e}}$, Gabriela A. Silva ${ }^{\mathrm{a}, \mathrm{f}, *}$ \\ a Centre for Biomedical Research (CBMR), University of Algarve, Faro 8005-139, Portugal \\ b Program in Biomedical Sciences, University of Algarve, Faro 8005-139, Portugal \\ c Department of Biomedical Sciences and Medicine, University of Algarve, Faro 8005-139, Portugal \\ d Department of Chemistry and Pharmacy, University of Algarve, Faro 8005-139, Portugal \\ e Algarve Chemistry Research Centre (CIQA), University of Algarve, Faro 8005-139, Portugal \\ f CEDOC, NOVA Medical School/Faculdade de Ciências Médicas, Universidade Nova de Lisboa, Campo Mártires da Pátria 130, 1169-056 Lisboa, Portugal
}

\section{A R T I C L E I N F O}

\section{Article history:}

Received 20 February 2015

Received in revised form 16 June 2015

Accepted 25 August 2015

Available online 28 August 2015

Keywords:

Gene therapy

Hyaluronic acid

Polymer modification

Retina

Biocompatibility

\begin{abstract}
A B S T R A C T
Purpose: A successful gene therapy approach can prevent or treat congenital and acquired diseases. However there is still no ideal non-viral vector for gene delivery in a safe and timely manner. In this report the anionic polymer hyaluronic acid (HA) was investigated as a potential vector for gene therapy. Due to its intrinsic characteristics it constitutes an excellent candidate to deliver therapeutic genes, pending the modification of its surface charge.

Methods: To modify its charge, HA was modified with cystamine. Several formulations were prepared using modified HA combined with sodium sulfate, sodium triphosphate, K-carrageenan and chitosan. Vectors were characterized with respect to size, charge, DNA load and its protection, and effect on cell viability. The better performing formulations were further evaluated in vitro for their transfection efficiency in HEK293T and ARPE-19 cells. Results: Cell viability assays showed low cytotoxicity for both polymers. Gene transfer efficiency depended on cell line and formulation, but no increased transfection efficiency was observed with the modified polymer. Conclusions: HA has great potential as a gene therapy vector, but further optimization, including incorporation of a higher percentage of positive groups in HA, is needed before its use as a gene delivery vector.
\end{abstract}

(c) 2015 Elsevier B.V. All rights reserved.

\section{Introduction}

Gene therapy is an area that has been growing rapidly and revolutionizing the world of research [1]. An essential requirement for the success of this technique is an efficient method for gene transfer, since DNA molecules cannot enter cells efficiently because of their large size, hydrophilic nature and susceptibility to degradation mediated by nucleases $[2,3]$. Considering the great diversity of diseases targeted by gene therapy, it is implausible that a single gene delivery system (vector) is suitable for all applications. However, the main requirements are common to all systems: vectors should transfer the genetic material to the tissue of interest and induce the proper level of therapeutic gene expression with no side effects [4].

Currently, vectors used in gene therapy can be divided into two main categories: viral and non-viral. Recombinant viruses such as retrovirus, lentivirus, adenovirus, adeno-associated viruses, and herpes viruses

Abbreviations: CS, chitosan; DNA, deoxyribonucleic acid; EDAC, 1-ethyl-3-(3dimethylaminopropyl)carbodiimide; HA, hyaluronic acid; $\mathrm{HASSNH}_{2}$, hyaluronic acid-co$\mathrm{N}$-cystaminyl-hyaluronamide; HASH, hyaluronic acid-co- $\mathrm{N}$-cysteaminyl-hyaluronamide; MW, molecular weight; RPE, retinal-pigmented epithelia.

* Corresponding author.

E-mail address: gabriela.silva@fcm.unl.pt (G.A. Silva). have been widely used for genetic material transfer [2]. Viruses have as main advantage their high transfection efficiency, however, viral vectors entail many intrinsic problems such as difficulties in production, limitations concerning repeated administrations that can lead to acute inflammatory responses, immune responses of the host to the virus and induction of mutagenesis by some viruses that integrate into the genome of host cells [2]. On the other hand, non-viral vectors have a higher safety profile due to their low toxicity and low immunogenicity. Additionally, non-viral vectors have the ability to carry larger genes and present lower production costs. Despite this appealing feature from the safety standpoint, non-viral vectors have little clinical importance due to low transfer and expression of transgene [2]. However, due to problems in clinical trials using viral vectors in the recent past, the interest in non-viral technologies has been renewed, particularly in the release properties of the non-viral vectors that resemble traditional drugs [4].

Non-viral vectors include synthetic or naturally occurring chemical compounds, such as lipids and cationic polymers. These can form complexes with the negatively charged DNA through electrostatic interactions that allow the therapeutic transgene across the cell membrane via endocytosis $[2,3,5]$. The complexes protect DNA from nucleasemediated degradation and facilitate cell entry as well as gene transferring into the nucleus [2]. 
Polymers have chemical flexibility, which enables the design and construction of polymers with multiple functionality, and thus more efficient in gene delivery, while maintaining the characteristics of biocompatibility, easy production and stable formulation [4]. One of the key points for the construction of more efficient vectors relates to various biological barriers of cells that must be overcome to achieve higher transfection rate. These barriers include the attachment of the polymer to the cell surface, then entering the cell through the cell membrane, displacement throughout the cytoplasm, escape endosomal degradation and ending with the passage of the nuclear envelope and nuclear entry [3]. In order to overcome different barriers, functional groups can be introduced into polymers, such as ligands to enhance cell entry via receptor-mediated endocytosis, membrane peptides to allow endosomal release and nuclear localization signals to increase nuclear entry of the transgene [3].

In this paper, hyaluronic acid (HA) was evaluated as a possible retinal gene therapy vector. HA is a biocompatible, non-toxic, nonimmunogenic, non-inflammatory anionic biopolymer that has been widely used in various biomedical applications [6]. Multiple studies on the biological function of HA have revealed that there is a strong relationship between the presence of HA and the migration and proliferation of cells as well as an involvement in wound healing, cell motility, angiogenesis, and extracellular matrix formation. Another important feature of HA for its use as a vector of therapeutic genes is the ability to interact with various cell receptors [6]. The negatively charged carboxyl group of HA is responsible for the interaction with membrane receptors allowing the connection with HA [7].

Despite its advantageous features, HA exists in the form of an aqueous gel, has a short lifetime and quickly degrades after administration. In order to increase the lifetime of HA for long-term clinical applications, several strategies have been developed, particularly modifications of the polymer at the level of carboxyl and hydroxyl groups. These new polymers, although they have different physico-chemical properties compared to unmodified HA, they maintain essential biological properties that allow their use in non-viral therapies [6]. Among possible chemical modifications to perform, the more relevant for gene therapy is the inclusion of amine groups [6]. A higher amount of amine groups may lead to greater DNA loads and increase the efficiency of transfection and transgene expression. In this study, HA was modified with cystamine through a coupling reaction with the carboxyl groups of HA. This modification with cystamine not only adds amine groups to the polymer but it also contains a disulfide bond that can be cleaved in the presence of intercellular glutathione, thereby promoting a more rapid release of genetic material $[8,9]$.

In this work we compare the transfection efficiency between modified and unmodified HA using two types of cells: human embryonic kidney (HEK 293T) cells and retinal pigment epithelium cells (ARPE-19). The former are a commonly used cell line in transfection studies, while the latter are a model of retinal pigmented epithelial (RPE) cells, our target in the retina, due to their important role in the support of the retinal homeostasis and involvement in several retinal diseases [8].

\section{Materials and methods}

\subsection{Materials}

Chitosan (MW of $80 \mathrm{kDa}$ ) with a degree of de-acetylation of $83 \%$ was purchased from Polysciences, Inc., USA. Hyaluronic acid, with MWs of 132 and 214 was purchased from Lifecore Biomedical. All other reagents were analytical grade and used without further purification.

\subsection{Plasmid constructs and cell lines}

A plasmid expressing enhanced green fluorescent protein driven by the cytomegalovirus promoter (kindly provided by Jean Bennett, University of Pennsylvania, USA) was amplified in Top 10 bacteria and purified using a Plasmid Maxi kit (Qiagen, California, USA) following manufacturer guidelines. Plasmid DNA (DNA) was dissolved in TE buffer, and the concentration was evaluated using a NanoDrop 2000c spectrophotometer (Thermo Scientific, Waltham, MA) at $260 \mathrm{~nm}$.

Two cell lines were used for transfection and cytotoxicity evaluation: HEK 293T cells (kindly provided by Guilherme Ferreira, University of Algarve, Portugal) and a human retinal pigment epithelial cell line (ARPE19, kindly provided by Francisco Ambrósio, University of Coimbra). All cell culture reagents were purchased from Sigma-Aldrich ${ }^{\circledR}$ (St. Louis, MO/USA).

\subsection{Methods}

\subsubsection{Modification of hyaluronic acid with cystamine $\left(\mathrm{HASSNH}_{2}\right)$}

The modification reaction was performed in an adaptation of what was previously described elsewhere and depicted in Fig. 1 [10-12]. Briefly, $500 \mathrm{mg}$ of hyaluronic acid was dissolved in $100 \mathrm{ml}$ of distilled $\mathrm{H}_{2} \mathrm{O}$, a one and a half molar excess (relative to the carboxylic acid groups in HA) of 1-ethyl-3-(3-dimethylaminopropyl)carbodiimide (EDAC, $0.378 \mathrm{~g}, 1.974 \mathrm{mmol})$, and three molar excess of cystamine $(0.889 \mathrm{~g}$, $3.947 \mathrm{mmol}$ ) were added to the solution. The mixture was stirred at room temperature for $72 \mathrm{~h}$. The reaction mixture was dialyzed against $4 \mathrm{~g} / \mathrm{l} \mathrm{NaCl}$ (MW cut off 2000) for $6 \mathrm{~h}$ and then against distilled $\mathrm{H}_{2} \mathrm{O}$ for $24 \mathrm{~h}$. The final product - $\mathrm{HASSNH}_{2}-$ was lyophilized for 3 days and stored at room temperature until use.

\subsubsection{Determination of the thiolation extent}

In order to determine the extent of the modification, the thiol groups were quantified by the Ellman's test. Firstly, $50 \mathrm{mg}$ of $\mathrm{HASSNH}_{2}$ was dissolved in distilled $\mathrm{H}_{2} \mathrm{O}$ and treated with 30 molar excess of dithiothreitol (DTT, $0.418 \mathrm{~g}, 2.712 \mathrm{mmol}$ ) in order to expose the thiol groups. The mixture was stirred under $\mathrm{N}_{2}$ atmosphere for $24 \mathrm{~h}$, at room temperature. The reaction mixture was dialyzed against $4 \mathrm{~g} / \mathrm{l} \mathrm{NaCl}$ (MW cut off 2000 ) for $6 \mathrm{~h}$ and then against distilled $\mathrm{H}_{2} \mathrm{O}$ for $24 \mathrm{~h}$. The final product HASH was lyophilized for 2 days and stored at room temperature until use. Secondly, for the Ellman's test, $3.8 \mathrm{mg}$ of HASH was dissolved in $1 \mathrm{ml}$ of $0.1 \mathrm{M}$ Tris buffer, $\mathrm{pH}$ 8.0. For each measurement, a tube containing $100 \mu$ l of Ellman's reagent ( $10 \mathrm{mM}$ of 5,5'-dithio-bis-(2-nitrobenzoic acid) in $0.1 \mathrm{M}$ Tris buffer), $1800 \mu \mathrm{l}$ of $0.1 \mathrm{M}$ Tris buffer and $100 \mu \mathrm{l}$ of sample was prepared, incubated at room temperature for $15 \mathrm{~min}$ and then the absorbance at $412 \mathrm{~nm}$ was measured [13].

\subsubsection{Potentiometric titration of $\mathrm{HASSNH}_{2}$}

In order to characterize the protonation behavior of the modified polymer, a titration was performed [14]. Polymer solutions, HA and $\mathrm{HASSNH}_{2}$, were prepared with a concentration of total titratable carbox$\mathrm{yl}$ group concentration of $10 \mathrm{mM}$ in a volume of $10 \mathrm{ml}$. The initial $\mathrm{pH}$ was adjusted to $2-3$ by adding $2.00 \mathrm{M} \mathrm{HCl}$ prior to the dropwise addition of the titrant $0.08 \mathrm{M} \mathrm{NaOH}$ to the polymer solutions under constant stirring. Potentiometric measurements were made using a $\mathrm{pH}$ measurement electrode (Orion 9157BN) connected to an Orion pH meter (Thermo Orion 4 Star pH-ISE Benchtop; ThermoFisher Scientific (Waltham, MA USA).

\subsubsection{Polyplex preparation}

Polyplexes were prepared as previously described by our lab $[8,15]$. Vector formulations were prepared at various ratios by adding anionic solutions to the $\mathrm{HASSNH}_{2}$ solution, as indicated in Diagram 1. Briefly, polymer solutions ( $1 \mathrm{mg} / \mathrm{ml}$ in MilliQ $\mathrm{H}_{2} \mathrm{O}, \mathrm{pH} 5.5$ ) and sodium sulfate solutions were separately heated to $55^{\circ} \mathrm{C}$. Equal volumes of both solutions were quickly mixed together, vortexed for $30 \mathrm{~s}$, placed on ice and stored at $4{ }^{\circ} \mathrm{C}$. Alternatively, HA and other anionic species (TPP and $\kappa$-carrageenan) were tested by combining them to the sodium sulfate solutions. Different weight ratios were tested keeping constant the $\mathrm{HASSNH}_{2}$ amount $(250 \mu \mathrm{g})$ and varying the HA amount. In order to use 


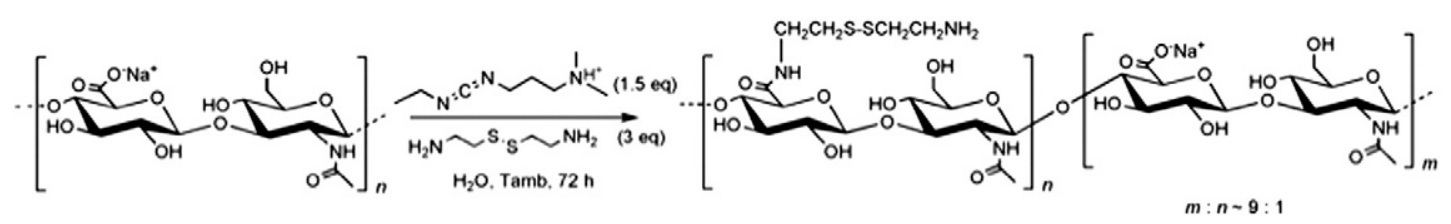

Fig. 1. Schematic representation of modification reaction and conditions.

equal volumes of both solutions, HA was diluted in sodium sulfate and then mixed with the $\mathrm{HASSNH}_{2}$ solution, as described above.

Vectors were prepared based on mixtures with chitosan (CS), originating CSHA or $\mathrm{CSHASSNH}_{2}$ vectors. The CS:HA or CS: $\mathrm{HASSNH}_{2}$ weight ratios 5:1 and 7:1 were chosen based on previous studies [15], keeping constant the CS amount ( $250 \mu \mathrm{g}$ ). In order to use equal volumes of both solutions, HA or $\mathrm{HASSNH}_{2}$ was diluted in $25 \mathrm{mM}$ sodium sulfate and then mixed with the CS solution, as described above.

To prepare polyplexes loaded with DNA at a $\mathrm{NH}_{3}^{+}: \mathrm{PO}_{4}^{-}$ratio of $15: 1$, $250 \mu \mathrm{g}$ of CS and $26.5 \mu \mathrm{g}$ of DNA, were used. DNA was mixed with the sodium sulfate solution and this solution mixed with the CS solution, as described above. The resulting polyplex formulations are described in Diagram 2.

\subsubsection{Polyplex characterization}

Size analysis was performed by dynamic light scattering (DLS) with a detection angle of $173^{\circ}$, and zeta potential (ZP) measured by laser Doppler velocimetry (Zetasizer Nano ZS, Malvern instruments, UK). All measurements were performed in $\mathrm{ddH}_{2} \mathrm{O}$ at $25^{\circ} \mathrm{C}$. The polydispersity index (PdI) was calculated based on DLS measurements using the Zetasizer Nano Series software version 7.11.

The complexation and integrity of the plasmids (DNA) in the polyplexes were assessed by gel electrophoresis. Free DNA and polyplexes were incubated with 1 unit of DNAse I (Sigma-Aldrich ${ }^{\circledR}$, USA) for $15 \mathrm{~min}$ at $37^{\circ} \mathrm{C}$. The reaction was stopped by the addition of $1 \mu \mathrm{l}$ of a $50 \mathrm{mM}$ EDTA solution and heating at $70^{\circ} \mathrm{C}$ for $10 \mathrm{~min}$. Complexation and integrity of the DNA were analyzed by agarose gel electrophoresis in $1 \%(\mathrm{~W} / \mathrm{V})$ agarose in TAE buffer with GreenSafe Premium (NZYTech, Portugal). Gels were subjected to a $90 \mathrm{mV}$ voltage for $1 \mathrm{~h}$ and further visualized under UV light (AlphaImager®, Alpha Innotech, USA).

Polyplex stability was also evaluated regarding physiological temperature $\left(37^{\circ} \mathrm{C}\right)$ and $\mathrm{pH}(7.4)$. Briefly, polyplexes were incubated in equal volumes of either PBS or DMEM (with 10\% FBS [fetal bovine serum]) at $37^{\circ} \mathrm{C}$ for 1 to 3 days. Polyplex stability, evaluated by DNA retention, was performed as described in the previous section.

To evaluate the cleavage of disulfide bonds incorporated into the modified polymer, polyplexes were incubated with glutathione and DTT. These assays were performed as described previously [8,9]. Briefly, a $0.4 \mathrm{M}$ glutathione reduced-form (Sigma) stock solution was prepared in $0.1 \mathrm{M}$ potassium phosphate buffer ( $\mathrm{pH} 7.5$ ), with 2 mM EDTA, pH 7.5 (potassium phosphate buffer with EDTA from here on referred to as PB). Polyplexes, prepared as previously described, were incubated for $24 \mathrm{~h}$ at $37^{\circ} \mathrm{C}$ in PBS in the presence of $20 \mathrm{mM}$ glutathione, 2 units $/ \mathrm{ml}$ of glutathione reductase (GlutR; Sigma), and $0.5 \mathrm{mM}$ of nicotinamide adenine dinucleotide phosphate (NADPH, Sigma). Polyplexes were incubated for $6 \mathrm{~h}$ at $37^{\circ} \mathrm{C}$ with different concentrations of DTT (10 and $\left.100 \mathrm{mM}\right)$ in sodium acetate at $10 \mathrm{mM}$. Samples were then analyzed by agarose gel electrophoresis as described in the previous section.

\subsubsection{In vitro assays}

Cells were cultured at $37^{\circ} \mathrm{C}$, under a $5 \% \mathrm{CO}_{2}$ atmosphere. Different culture media were used, according to each cell's specifications: HEK293T in Dulbecco's Modified Eagle's Medium (DMEM) and ARPE19 in DMEM mixture with F-12 HAM; both supplemented with $10 \%$ of fetal bovine serum, $1 \%$ penicillin/streptomycin and $1 \%$ glutamine.

An MTT assay was performed to evaluate the cytotoxicity of the polymers. Cells were seeded at a density of $1.5 \times 10^{4}$ cells/well in 48 well flat bottom tissue culture plates and the assay was carried out as

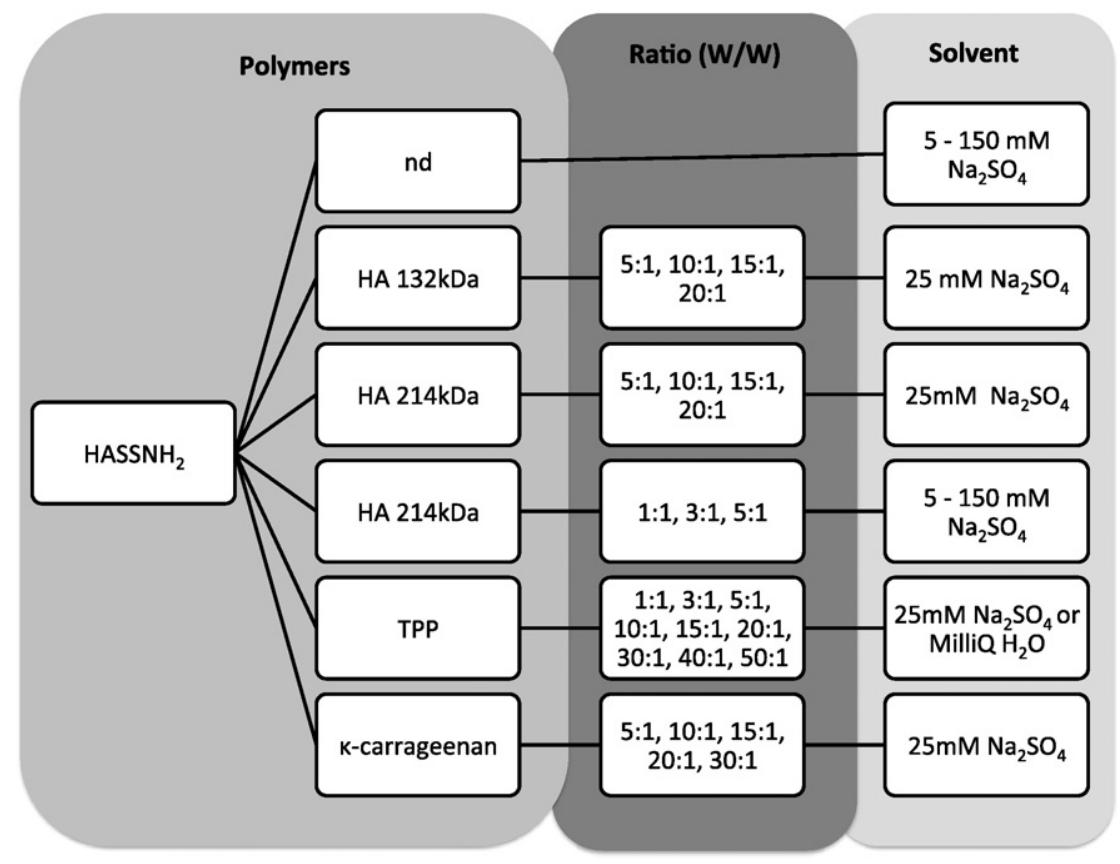

Diagram 1. Tested vector formulations with different anionic agents and different weight ratios, nd-not determined. 


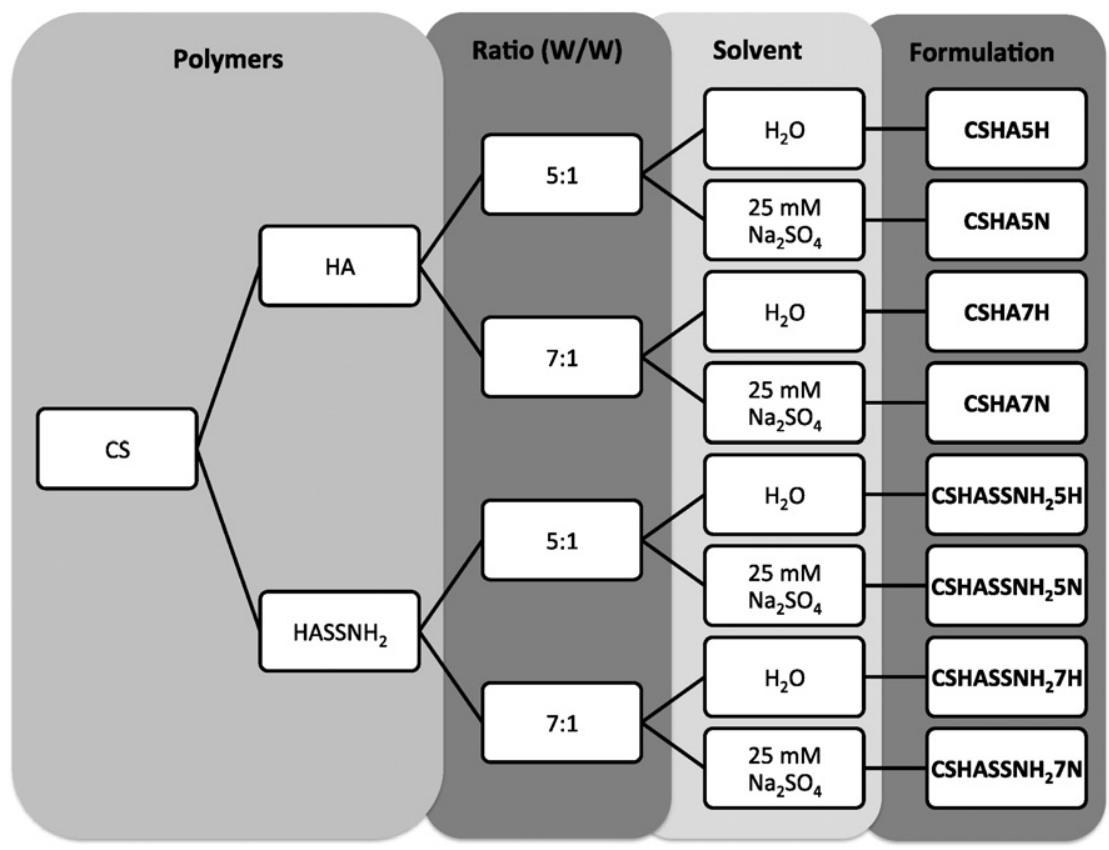

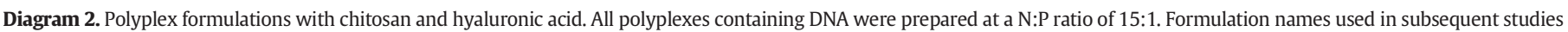
are shown in bold.

described previously [8]. Cells were incubated in culture medium containing different amounts of polymer (from $0.01 \mathrm{up}$ to $0.1 \mathrm{mg} / \mathrm{ml}$ ) for 24 and $72 \mathrm{~h}$. As positive and negative controls of cell viability, cells cultured in standard cell culture conditions and cells incubated with a latex extract in culture medium $\left(1.5 \mathrm{~cm}^{2} / \mathrm{ml}\right)$ were used, respectively. Absorbance was measured using a microplate reader (Tecan Infinite 2000, USA), at 570 and $630 \mathrm{~nm}$, for cell viability/formazan formation and background, respectively. Cell viability was calculated as follows: Cell viability $(\%)=($ ODsample $) /($ ODcontrol $) \times 100$, where ODcontrol and ODsample are cells not challenged and challenged by polyplexes, respectively. Each value was averaged from triplicates and each experiment was carried out thrice.

For the transfection studies, cells were seeded at $2 \times 10^{5}$ cells/well in 6 -well tissue culture plates $24 \mathrm{~h}$ prior to the transfection. FuGENE® HD (Promega, USA) was used as positive transfection control according to the manufacturer's instructions. Polyplexes were added to cells at a ratio of $1 \mu \mathrm{g}$ of DNA per well and further incubated for $5 \mathrm{~h}$ in serumfree media. Then, media was replaced by complete media and transfection efficiency was evaluated quantitatively $72 \mathrm{~h}$ post-transfection by flow cytometry by scoring GFP-positive cells (FACScalibur, BD Biosciences, USA) using FL- $1 \mathrm{H}$, green channel. A total of $5 \times 10^{4}$ events were counted for each sample. The percentage of positive events corresponds to the gated events minus the non-transfected cells. Transfection results were also evaluated qualitatively by fluorescence microscopy (Axiovert 40 CFL, Zeiss).

\subsubsection{Statistical analysis}

Results presented are mean \pm standard deviation of at least three independent experiments. Statistical analysis was performed with GraphPad Prism version 6.04 software. Data were subjected to analysis of variance and multiple comparisons tests using a confidence interval of $95 \%$.

\section{Results}

\subsection{Characterization of the modified polymer: degree of modification}

The first step in the characterization of the polymer was to determine the extent of modification by quantification of the added thiol groups. After treatment with an excess of DTT it was possible to quantify the thiol groups present in the polymer through Ellman's test. The average percentage of thiol substitution to the total carboxylic acid groups of HA was $9.88 \% \pm 0.34$.
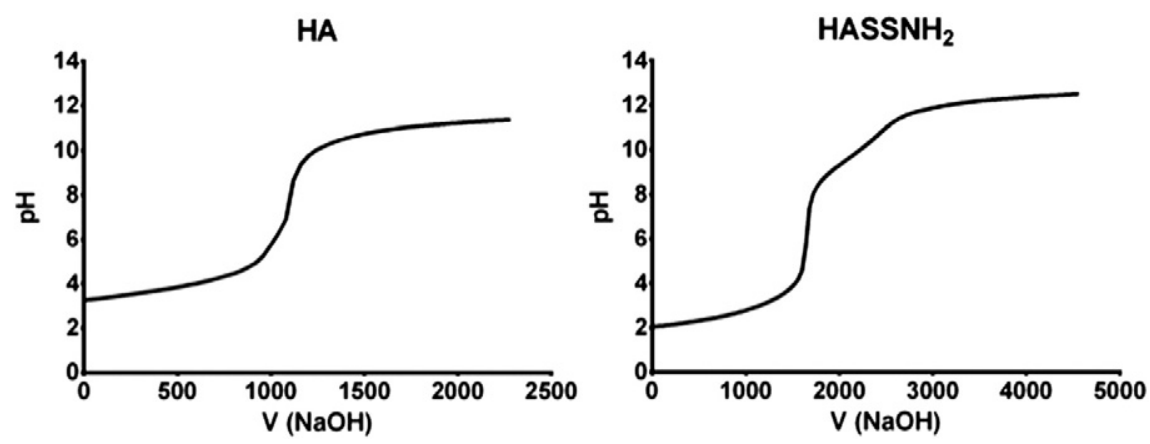

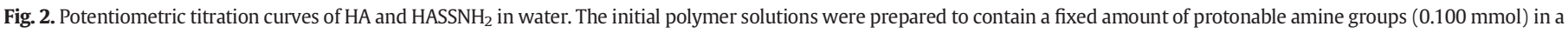

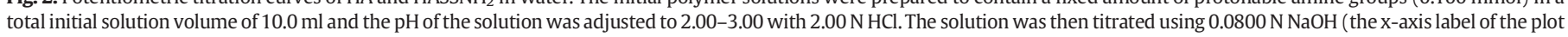
denotes the total volume of added $\mathrm{NaOH}$ in $\mu \mathrm{l}$ ). 

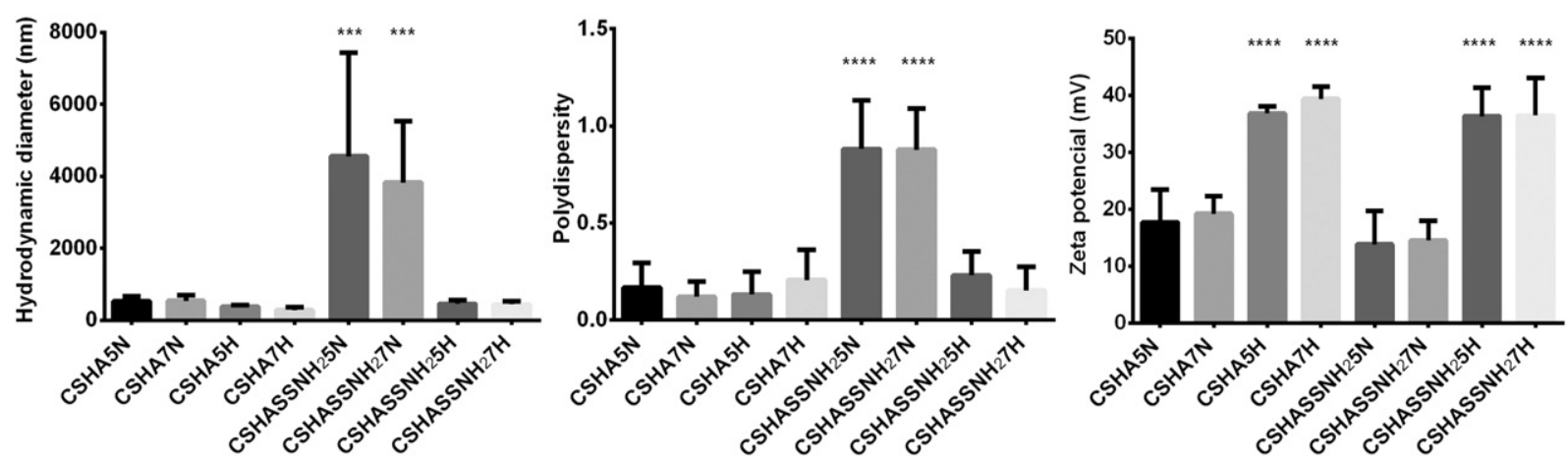

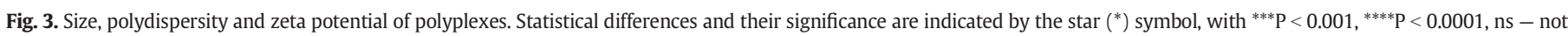
significant.

The behavior of $\mathrm{HASSNH}_{2}$ as a function of $\mathrm{pH}$ was then evaluated by titration, where the carboxylic group $\left(\mathrm{COO}^{-}\right)$was considered to be the protonatable group. Comparing the unmodified and modified titration curves it is possible to observe differences in the equivalence points present confirming the success of the modification reaction (Fig. 2).

\subsection{Physical characterization of $\mathrm{HASSNH}_{2}$ polyplexes}

Several conditions were tested in order to produce vectors formulations with adequate characteristics. Firstly, formulations based on $\mathrm{HASSNH}_{2}$ and different concentrations of anionic agents were tested $\left(\mathrm{Na}_{2} \mathrm{SO}_{4}\right.$, TPP, HA and $\kappa$-carrageenan) but did not yield polyplexes with the appropriate size $(<500 \mathrm{~nm})$ or polydispersity $(<0.5$, data not shown). Then, formulations with the cationic polymer chitosan and the modified polymer or HA were examined, yielding positive results as described in Fig. 3. Only formulations $\mathrm{CSHASSNH}_{2} 5 \mathrm{~N}$ and $\mathrm{CSHASSNH}_{2} 7 \mathrm{~N}$ presented size and polydispersity above average. Regarding polyplexes prepared in water (CSHA5H, CSHA7H, $\mathrm{CSHASSNH}_{2} 5 \mathrm{H}$ and $\mathrm{CSHASSNH}_{2} 7 \mathrm{H}$ ) these presented much higher zeta potential values with approximately a 2 -fold increase in the surface charge.

Next, polyplexes were evaluated regarding their DNA complexing capacity and nuclease protection. Polyplexes were capable of efficient DNA complexion and protection against DNAse-induced degradation, as verified by agarose gel retardation assays (Fig. 4). Also, polyplexes remained stable and did not release DNA in detectable amounts, even after incubation for several days at physiological conditions. However, upon incubation in media with serum in some of the formulations it is possible to observe some DNA release (Fig. 5) indicating a less stable polyplex. Nevertheless it is noteworthy that the DNA released by the polyplexes is still undamaged whereas the free DNA used as control is clearly degraded indicating the polyplexes did protect the DNA.

In order to evaluate the effect of a reductive environment on polyplex DNA release, polyplexes were incubated in the presence of glutathione and DTT and DNA release was subsequently analyzed by gel retardation assays. No detectable amount of DNA was released from the polyplexes in either condition, as observed in Fig. 6.

\subsection{In vitro studies: cytotoxicity and transfection}

The cytotoxicity evaluation was performed by an MTT assay to study possible changes in the cytocompatibility of the modified polymer by comparison with the unmodified HA. The results are presented in Fig. 7, and show no differences both for tested concentrations and polymers.

After verifying that there were no cytotoxicity associated with the polymers, the polyplexes were evaluated regarding their transfection efficiency in two cell lines, HEK293T and ARPE-19, the first are a commonly used cell line in transfection studies, while the latter are a model of retinal pigment epithelial (RPE) cells, our target in the retina.

In ARPE-19 cells no differences between different formulations were found whereas in HEK293T there was statistically lower transfection efficiency when $\mathrm{HASSNH}_{2}$ polyplexes were prepared in sodium sulfate (Fig. 8). Nonetheless, polyplexes prepared in water, with both HA and $\mathrm{HASSNH}_{2}$, had similar transfection results (Figs. 8 and 9).

\section{Discussion}

\subsection{HA was successfully modified with cystamine}

Hyaluronic acid was modified by reaction of the carboxylic groups of HA with the amine groups of cystamine dihydrochloride using EDAC activation. Later, a portion of the modified polymer was treated using an excess amount of the DTT to cleave the disulfide bonds. The resulting product (HASH) was purified by dialysis and isolated after freezedrying. The average percentage of thiol substitution (approximately $10 \%$ ) was determined by Ellman's test. This percentage of substitution is in agreement with values in the literature since it is considered that a partial modification (10-20\%) is desirable to achieve polyplexes with well-defined sizes [10].
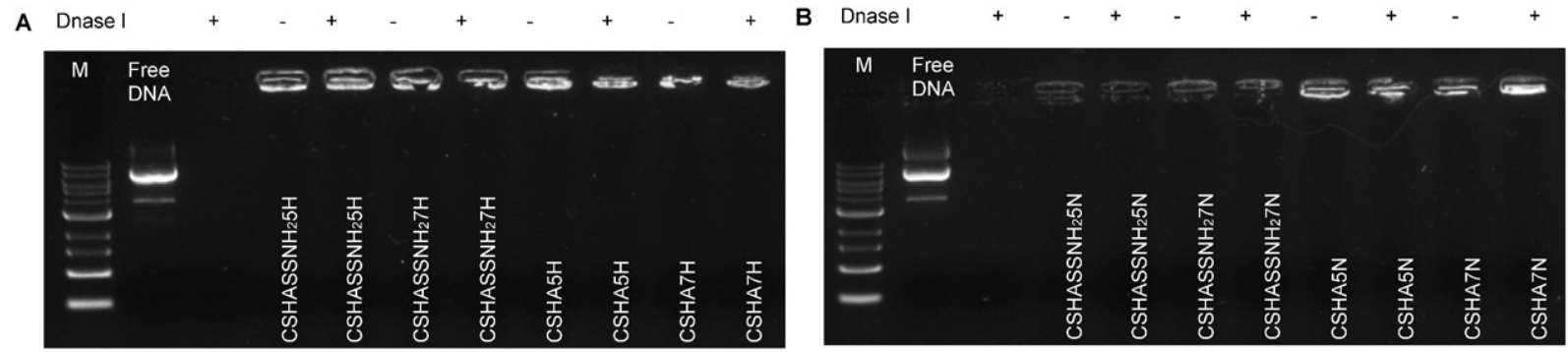

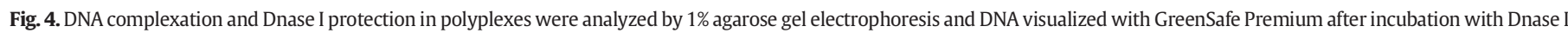
for 15 min at $37{ }^{\circ} \mathrm{C}(+)$ for formulations $\mathrm{A}$ ) in $\mathrm{H}_{2} \mathrm{O}$ and $\mathrm{B}$ ) in $\mathrm{Na}_{2} \mathrm{SO}_{4}$. 


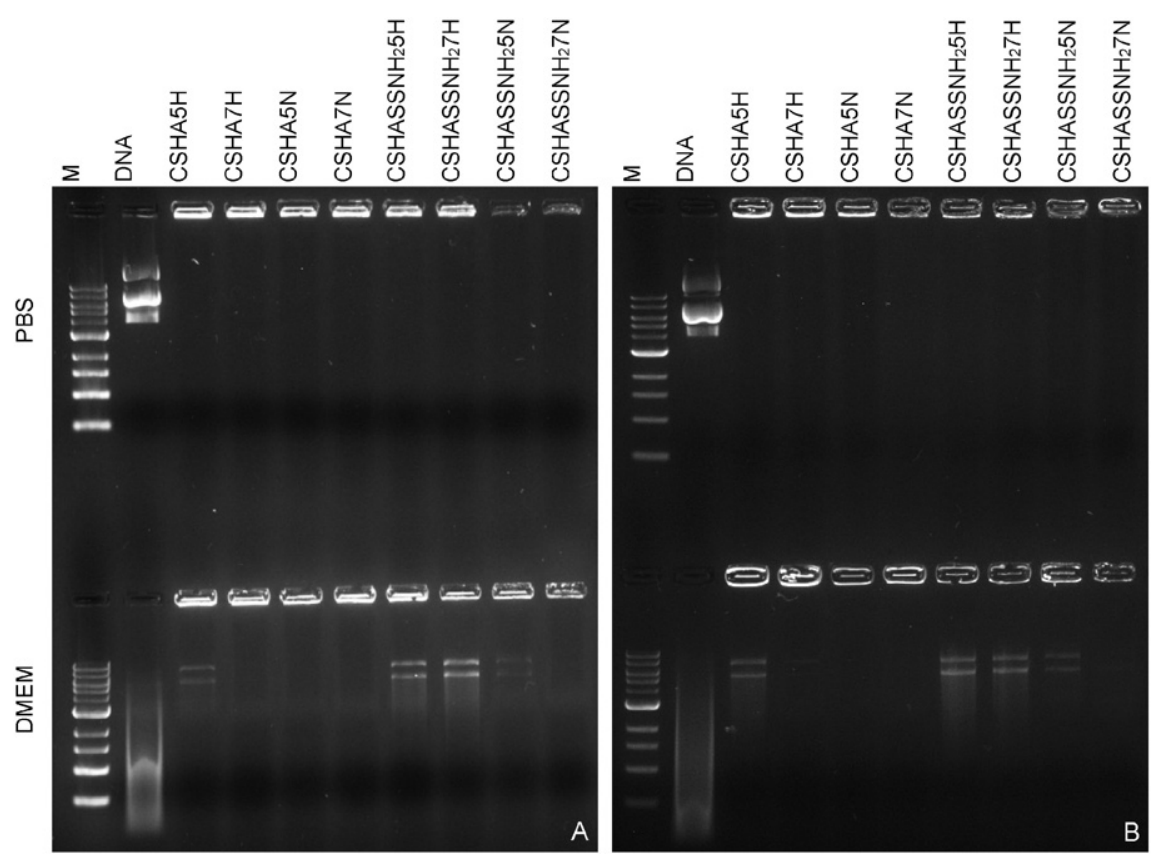

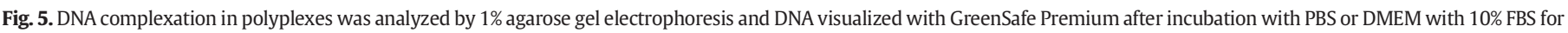
A) 3 and B) 7 days at $37^{\circ} \mathrm{C}$.

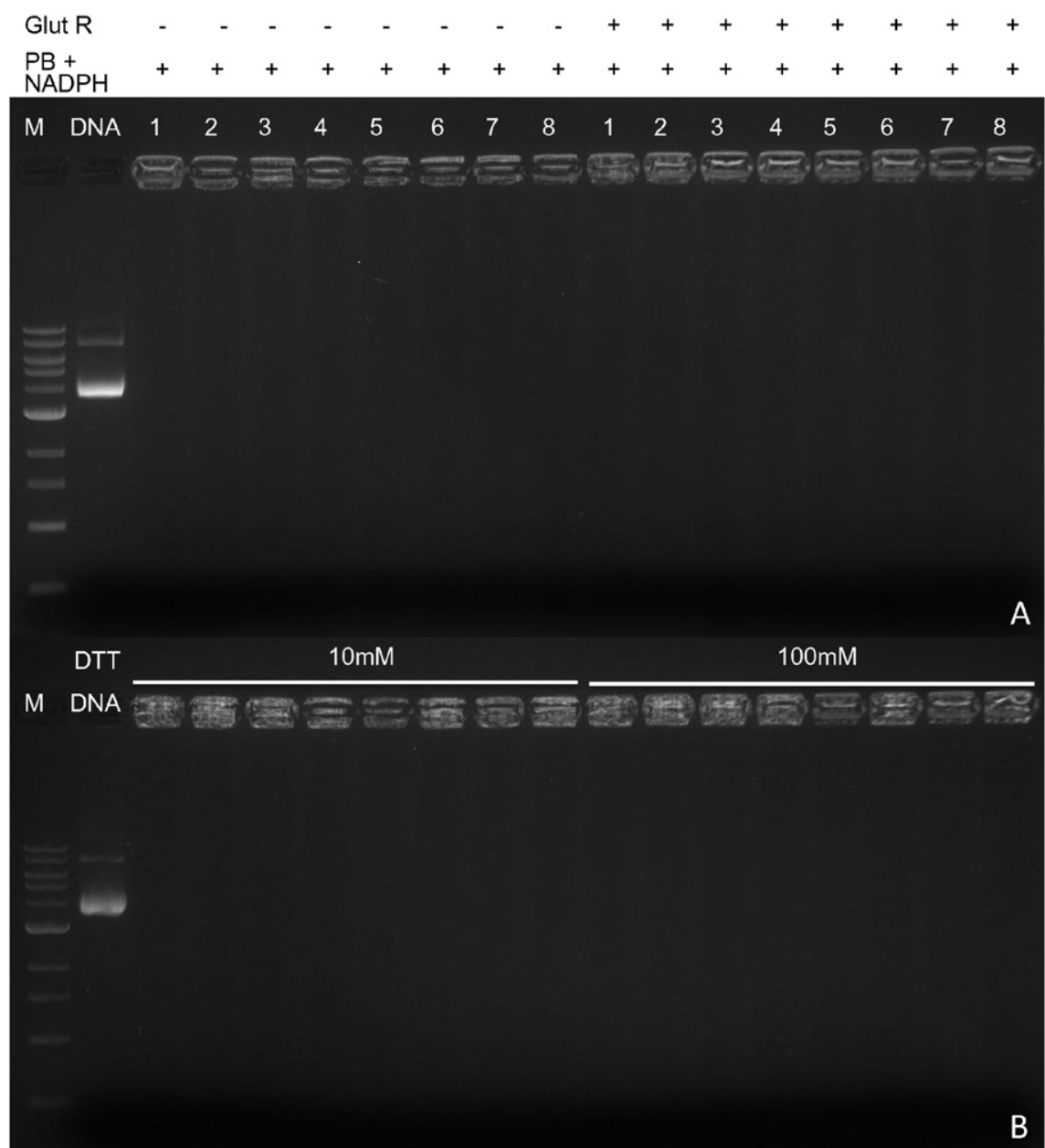

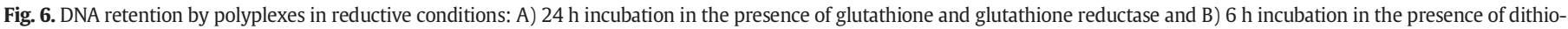
threitol (DTT); polyplexes in lanes: 1-CSHA5N, 2-CSHA5H, 3-CSHA7N, 4-CSHA7H, 5-CSHASSNH ${ }_{2} 5 \mathrm{~N}^{2} 6-\mathrm{CSHASSNH}_{2} 5 \mathrm{H}, 7-\mathrm{CSHASSNH}_{2} 7 \mathrm{~N}, 8-\mathrm{CSHASSNH}_{2}$ 7H. 

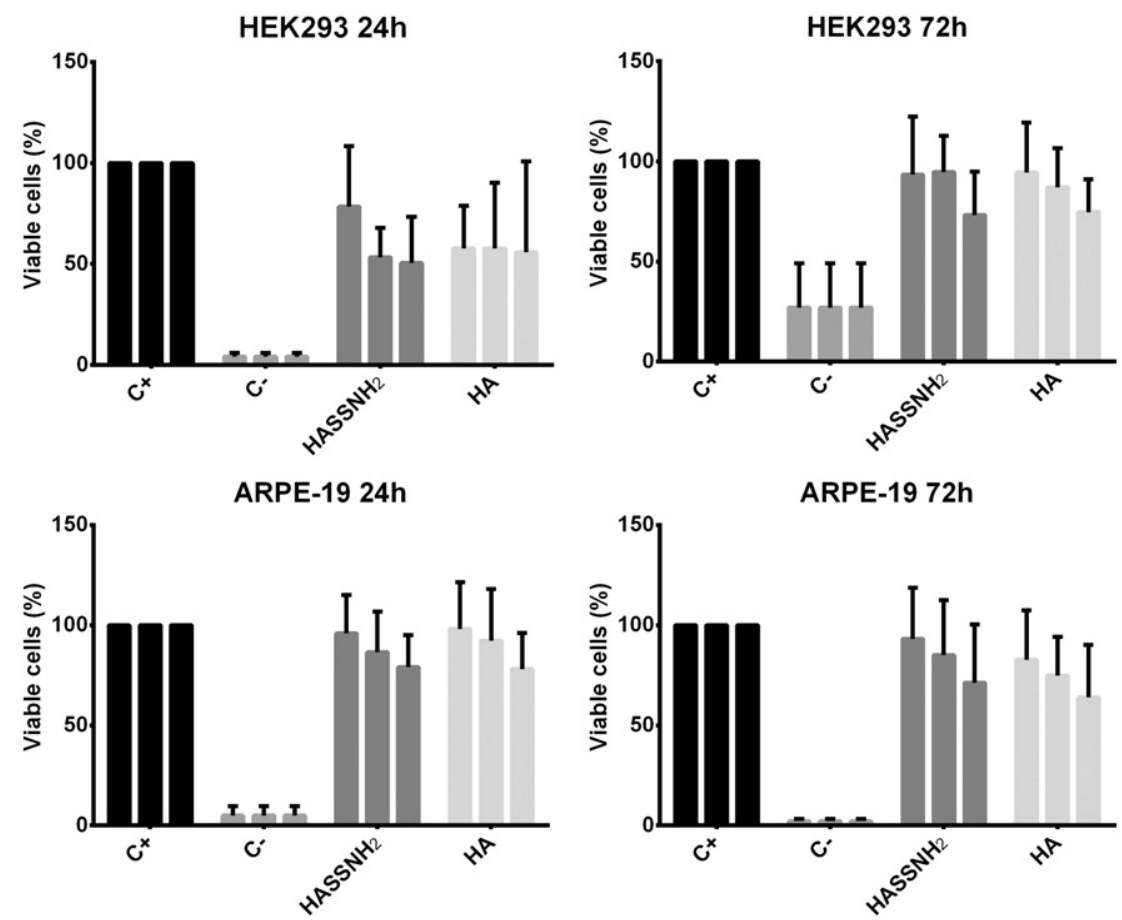

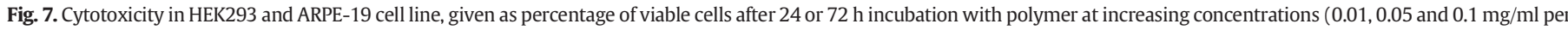
well). Un-challenged cells and cells incubated with latex extracts were used as positive and negative controls of cellular viability.

The presence of two equivalence points in the potentiometric titration curve of $\mathrm{HASSNH}_{2}$ further confirmed the success of the modification reaction and indicated the $\mathrm{pH}$ range more adequate for polyplex preparation. At $\mathrm{pH}$ values much below the first equivalence point the carboxyl groups are predominantly deprotonated. This may hinder the preparation of polyplexes because in this case carboxyl groups may interact with amine groups in the polymer, leaving very few amine groups available to interact with DNA phosphate groups. On the other hand, at $\mathrm{pH}$ values exceeding the second equivalence point all amine groups are deprotonated and there are fewer positive charges available to interact with DNA phosphate groups, establishing less electrostatic interactions. Thus it is preferable to prepare polyplexes at pH values between 6 and 9 , which is also desirable for later in vitro and in vivo experiments.

\section{2. $\mathrm{HASSNH}_{2}$ does not produce polyplexes with anionic agents}

Initially nanoparticles were evaluated and characterized with respect to their size and polydispersion. Several formulations with $\mathrm{HASSNH}_{2}$ and different concentrations of anionic agents were tested
( $\mathrm{Na}_{2} \mathrm{SO}_{4}$, TPP, HA and $\kappa$-carrageenan) with no satisfactory results. $\mathrm{HASSNH}_{2} /$ DNA polyplexes were also tested at various N:P ratios but complete complexation was not achieved (data not shown). The modified polymer was unable to generate polyplexes by itself or with the addition of other anionic agents, probably due to insufficient electrostatic interactions. This may be related to the degree of substitution of the modified polymer that seems to have a low number of positive charges available to interact. In order to increase the number of positive charges and foster electrostatic interactions, chitosan was added to the formulation yielding the results described in the next section.

\subsection{CSHA and $\mathrm{CSHASSNH}_{2}$ polyplexes: a comparison}

Previous studies in our lab had shown positive results in terms of transfection with formulations based on chitosan and hyaluronic acid [15]. In this study we wanted to evaluate and compare the performance of polyplexes prepared with normal unmodified hyaluronic acid and polyplexes prepared with $\mathrm{HASSNH}_{2}$. Different formulations were prepared and the first step in their evaluation was to characterize them
HEK293T

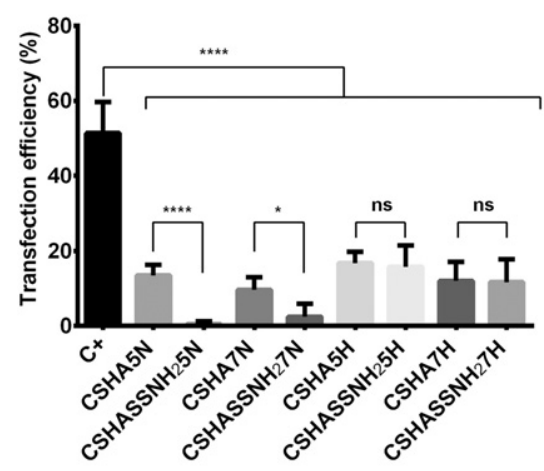

ARPE-19

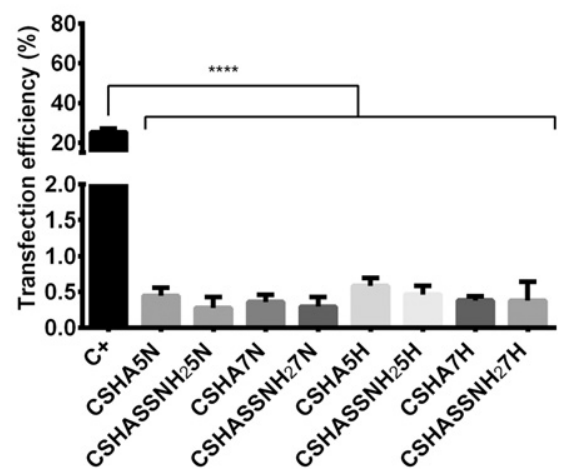

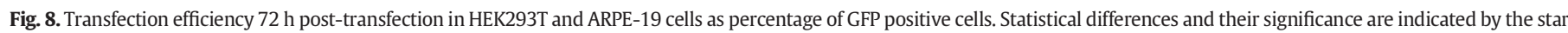
(*) symbol, with ${ }^{*} \mathrm{P}<0.05,{ }^{* * * *} \mathrm{P}<0.001$, ns - not significant. 

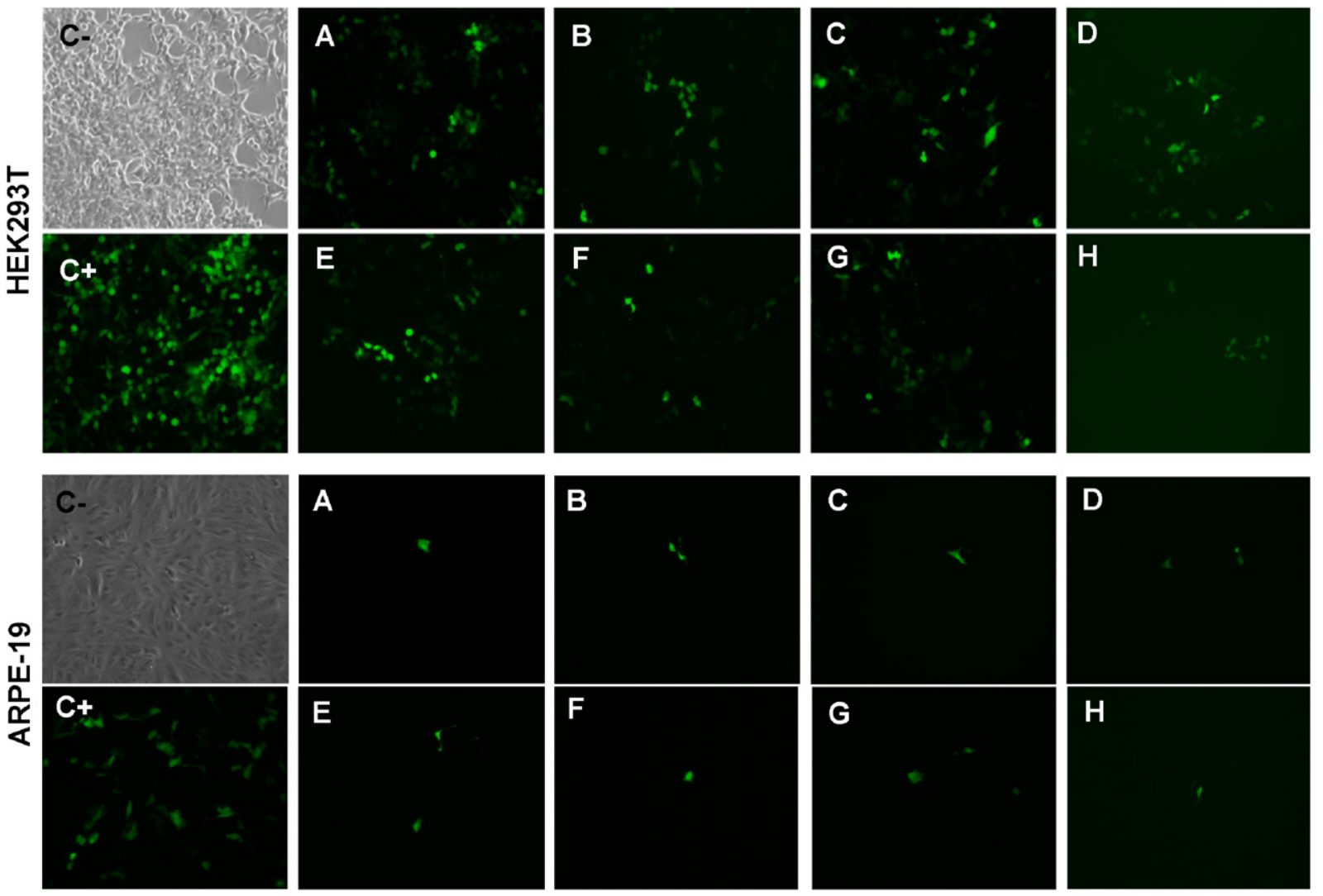

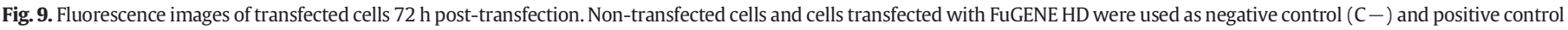

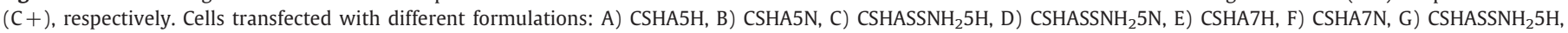
H) $\mathrm{CSHASSNH}_{2} 5 \mathrm{~N}$. Total amplification $100 \times$.

for their size, polydispersity, and zeta potential (Fig. 3). Polyplexes were compared through a statistical multiple comparison analysis. Among the various comparisons, those with higher significance for the interpretation of the results were comparisons 1 ) between formulations with different solvents but the same polymer and ratio; 2 ) between formulations with different ratios while maintaining the same polymer; and 3 ) between formulations with different polymers but keeping the same ratio and solvent. Given the comparisons described above, in Fig. 3 are represented all comparisons with a significant statistical difference. All other comparisons show no statistical difference.

The different formulations yielded polyplexes with similar sizes except for $\mathrm{CSHASSNH}_{2} 5 \mathrm{~N}$ and $\mathrm{CSHASSNH}_{2} 7 \mathrm{~N}$. These two formulations also presented high polydispersity and a slightly lower surface charge (given by the zeta potential values). The zeta potential can be used to predict the stability of the dispersion so this reduced surface charge might have caused aggregation of the polyplexes hence the large size and polydispersity [16]. Since chitosan is a polycation, a positive zeta potential was expected, which occurred in all cases. However, statistical differences were observed between polyplexes with the same polymer and ratio but different solvents $\left(\mathrm{Na}_{2} \mathrm{SO}_{4}\right.$ and $\left.\mathrm{H}_{2} \mathrm{O}\right)$. This may be due to the presence of the negative charges from sulfate anions, which reduce the overall surface charge.

\subsection{Polymer modification did not affect cytotoxicity}

The cell viability assay main objective was to evaluate the possible cytotoxic effect of the polymers in the cells after DNA release and to assess if the modification changed the polymer cytotoxicity profile. HA is known as a biocompatible polymer and studies have shown that it is safe for concentrations up to $1-2 \%(\mathrm{~W} / \mathrm{V})[17,18]$. Comparing the results obtained for the HA and $\mathrm{HASSNH}_{2}$ polymers, no statistical differences were observed in all four tested situations. This is possibly due to the fact that the difference in composition of the two polymers is small, since only $10 \%$ modification was achieved, and this did not sufficiently alter the toxicity profile of the polymer.

However, after $24 \mathrm{~h}$ of incubation $\mathrm{HA}$ and $\mathrm{HASSNH}_{2}$ showed a reduction of cell viability in HEK293 cells for all tested concentrations when compared to the control. This was not observed for the other tested conditions and this could be related to the fact that at $24 \mathrm{~h}$ the cells underwent acute exposure while at $72 \mathrm{~h}$ it resembles a chronic exposure. The fact that the cell viability had decreased slightly in some cases may also be related to the fact that the polymer solutions have not been extensively purified before addition to cells, and so the observed cytotoxicity may be due to residual impurities of the modified polymer. Nevertheless, at concentrations to be used in future in vitro and in vivo transfection studies, no cytotoxicity was observed.

\subsection{Polyplex stability at physiological conditions varies with temperature and presence of serum}

All polyplexes were capable of efficient DNA complexion and protection against DNAse-induced degradation when exposed to DNase I (Fig. 4 ). This is a critical aspect for all non-viral vectors, since a successful gene therapy vector needs to protect its load until it reaches the target and only unload it at the appropriate time [19,20]. Our stability assay results shown that all polyplexes remained stable up to 7 days at physiological temperature and $\mathrm{pH}$ (polyplexes incubated with PBS). However, when polyplexes were incubated in DMEM with serum some DNA release was observed, particularly in polyplexes with the modified polymer. It is hypothesized that serum components establish electrostatic interactions with the polyplexes, destabilizing them, and causing DNA release. The fact that polyplexes prepared with the modified polymer appear less stable can actually be advantageous since several studies 
have shown a negative correlation between polyplex stability and transfection efficiency [15,21-23].

\subsection{Transfection efficiency is formulation-dependent}

One of the main objectives of this study was to evaluate if the modification of HA would affect the stability of the polyplexes and consequently their transfection efficacy. In our study, different formulations of $\mathrm{CSHASSNH}_{2}$ polyplexes showed very similar transfection efficiencies. Analyzing the results obtained for ARPE-19 and HEK293 cells, the latter had much higher transfection efficiency in all formulations due to their higher permissibility to transfection, associated with their higher mitotic rate. Despite the higher transfection values there were only differences in the transfection efficiency between CSHA and $\mathrm{CSHASSNH}_{2}$ polyplexes prepared in sodium sulfate. These polyplexes ( $\mathrm{CSHASSNH}_{2} 5 \mathrm{~N}$ and $\mathrm{CSHASSNH}_{2} 7 \mathrm{~N}$ ) were expected to have a reduced efficacy since their size was too large for an efficient and timely cellular internalization and this was indeed confirmed.

Moreover, less stable formulations, with visible DNA release in the stability assay, displayed higher transfection values as expected, based on the aforementioned relation between polyplex stability and transfection efficiency.

Polyplex charge is a key parameter due to its association with toxicity and transfection efficiency. Other studies have shown an association between surface charge and increased transfection efficiency as well as a decrease in toxicity $[24,25]$. Also, cellular uptake is one of the major obstacles for a successful gene delivery strategy and polyplexes are thought to enter the cell via endocytosis in a process that may be dependent on polyplex charge [26]. A higher surface charge can facilitate electrostatic interactions with the negatively charged proteoglycans in cellular membranes and hence promote polyplex internalization [27]. Our results show a positive correlation between polyplex surface charge and transfection efficiency that is in agreement with the literature. Polyplexes prepared in water displayed nearly a 2-fold increase in surface charge that correlated with higher transfection values, irrespective of the polymer used. This data strongly indicates that the preparation method used for polyplex formation is as important as the polymer used.

\section{Conclusions}

This work had as main objective the characterization of a novel polymer, $\mathrm{HASSNH}_{2}$, for subsequent use as a retinal gene therapy vector. After confirming the success of the modification reaction and determining its extent $(\sim 10 \%)$, several strategies were tested in order to yield polyplexes with adequate characteristics. The resulting polyplexes were capable of effective DNA complexation though their stability at physiological conditions varied. Transfection studies using HEK293 and ARPE-19 cells showed relatively modest transfection efficiency where no differences were observed between polyplexes with modified or unmodified HA. Higher transfection values correlated with lower polyplex stability as well as higher surface charge.

In the future, higher degrees of modification will be pursued by introducing changes in the reaction conditions, in order to modulate the stability and DNA release from the polyplexes, to allow further improvement of the transfection efficiency of these gene therapy vectors.

\section{Acknowledgments}

The authors acknowledge the financial support of Fundação para a Ciência e Tecnologia (PTDC/SAU-BEB/098475/2008 to Gabriela A. Silva, SFRH/BD/70318/2010 individual fellowship to Ana V. Oliveira, IBB/LA under the project PEst-OE/EQB/LA0023/2013, and PEst-
OE_QUI_UI4023_2011) and the Marie Curie Reintegration Grant (PIRG-GA-2009-249314 to Gabriela A. Silva) under the FP7 program.

\section{References}

[1] P.D. Robbins, S.C. Ghivizzani, Viral vectors for gene therapy, Phamacological Therapy 80 (1998) 35-47.

[2] M.S. Al-Dosari, X. Gao, Nonviral gene delivery: principle, limitations, and recent progress, AAPS J. 11 (2009) 671-681.

[3] I.A. Khalil, Uptake pathways and subsequent intracellular trafficking in nonviral gene delivery, Pharmacol. Rev. 58 (2006) 32-45.

[4] D.C. Gorecki, "Dressed-up" naked plasmids: emerging vectors for non-viral gene therapy, Discovery Medicine 6 (2006) 191-197.

[5] I.M. Verma, N. Somia, Gene therapy - promises, problems and prospects, Nature 389 (1997) 4.

[6] E.J. Oh, K. Park, K.S. Kim, J. Kim, J.-A. Yang, J.-H. Kong, et al., Target specific and longacting delivery of protein, peptide, and nucleotide therapeutics using hyaluronic acid derivatives, J. Control. Release 141 (2010) 2-12.

[7] R.J. Peach, D. Hollenbaugh, I. Stamenkovic, A. Aruffo, Identification of hyaluronic acid binding sites in the extracellular domain of CD44, J. Cell Biol. 122 (1993) 257-264.

[8] A.V. Oliveira, A.P. Silva, D.B. Bitoque, G.A. Silva, A.M. Rosa da Costa, Transfection efficiency of chitosan and thiolated chitosan in retinal pigment epithelium cells: a comparative study, Journal of Pharmacy \& Bioallied Sciences 5 (2013) 111-118.

[9] C. Pichon, E. LeCam, B. Guérin, D. Coulaud, E. Delain, P. Midoux, Poly[Lys-(AEDTP)]: a cationic polymer that allows dissociation of pDNA/cationic polymer complexes in a reductive medium and enhances polyfection, Bioconjug. Chem. 13 (2002) 76-82.

[10] R. Jin, L.S. Moreira Teixeira, A. Krouwels, P.J. Dijkstra, C.A. van Blitterswijk, M Karperien, et al., Synthesis and characterization of hyaluronic acid-poly(ethylene glycol) hydrogels via Michael addition: an injectable biomaterial for cartilage repair, Acta Biomater. 6 (2010) 1968-1977.

[11] H. Lee, S.H. Choi, T.G. Park, Direct visualization of hyaluronic acid polymer chain by self-assembled one-dimensional array of gold nanoparticles, Macromolecules 39 (2006) 23-25.

[12] H. Lee, H. Mok, S. Lee, Y.-K. Oh, T.G. Park, Target-specific intracellular delivery of siRNA using degradable hyaluronic acid nanogels, J. Control. Release 119 (2007) $245-252$.

[13] G.L. Ellman, K.D. Courtney, J. Valentino Andres, R.M. Featherstone, A new and rapid colorimetric determination of acetylcholinesterase activity, Biochemical Pharmacological 7 (1961) 88-95.

[14] D.B. Bitoque, S. Simao, A.V. Oliveira, S. Machado, M.R. Duran, E. Lopes, et al., Efficiency of RAFT-synthesized PDMAEMA in gene transfer to the retina, Journal of Tissue Engineering and Regenerative Medicine (2014)http://dx.doi.org/10.1002/term. 1909 (in press).

[15] Oliveira AV, Bitoque DB, Silva GA. Combining hyaluronic acid with chitosan enhances gene delivery. J. Nanomater. 2014;2014:9.

[16] Nobbmann U. Protein sizing by light scattering, molecular weight and polydispersity. In: Instruments M, editor. http://www.malvern.co.uk/proteins.

[17] D.G. Boeckel, R.S. Shinkai, M.L. Grossi, E.R. Teixeira, In vitro evaluation of cytotoxicity of hyaluronic acid as an extracellular matrix on OFCOL II cells by the MTT assay, Oral Surgery, Oral Medicine, Oral Pathology and Oral Radiology 117 (2012) e423-8.

[18] Becker LC, Bergfeld WF, Belsito DV, Klaassen CD, Jr JGM, Shank RC, et al. Final report of the safety assessment of hyaluronic acid, potassium hyaluronate, and sodium hyaluronate. Int. J. Toxicol. 2009;28:5-67.

[19] S. Danielsen, S. Strand, D.C. de Lange, B.T. Stokke, Glycosaminoglycan destabilization of DNA-chitosan polyplexes for gene delivery depends on chitosan chain length and GAG properties, Biochim. Biophys. Acta 2005 (1721) 44-54.

[20] M. Koping-Hoggard, K.M. Varum, M. Issa, S. Danielsen, B.E. Christensen, B.T. Stokke et al., Improved chitosan-mediated gene delivery based on easily dissociated chitosan polyplexes of highly defined chitosan oligomers, Gene Ther. 11 (2004) 1441-1452.

[21] X. Zhao, S.-B. Yu, F.-L. Wu, Z.-B. Mao, C.-L. Yu, Transfection of primary chondrocytes using chitosan-pEGFP nanoparticles, J. Control. Release 112 (2006) 223-228.

[22] T. Kiang, J. Wen, H.W. Lim, K.W. Leong, The effect of the degree of chitosan deacetylation on the efficiency of gene transfection, Biomaterials 25 (2004) 5293-5301.

[23] N. Duceppe, M. Tabrizian, Factors influencing the transfection efficiency of ultra low molecular weight chitosan/hyaluronic acid nanoparticles, Biomaterials 30 (2009) 2625-2631.

[24] P. Erbacher, S. Zou, T. Bettinger, A.M. Steffan, J.S. Remy, Chitosan-based vector/DNA complexes for gene delivery: biophysical characteristics and transfection ability, Pharm. Res. 15 (1998) 1332-1339.

[25] C. Perez, A. Sanchez, D. Putnam, D. Ting, R. Langer, M.J. Alonso, Poly(lactic acid)poly(ethylene glycol) nanoparticles as new carriers for the delivery of plasmid DNA, J. Control. Release 75 (2001) 211-224.

[26] T. Ishii, Y. Okahata, T. Sato, Mechanism of cell transfection with plasmid/chitosan complexes, Biochimica et Biophysica Acta (BBA) 1514 (2001) 51-64.

[27] M. Agirre, J. Zarate, G. Puras, E. Ojeda, J.L. Pedraz, Improving transfection efficiency of ultrapure oligochitosan/DNA polyplexes by medium acidification, Drug Delivery 22 (2015) 100-110. 